\title{
AVRS for Parent of Students using Rasp Pi
}

\author{
Jagruti Patil \\ ME Scholar \\ Dept. of E\&TC \\ GF's GCOE, Jalgaon, India
}

\author{
V.D. Chaudhari \\ Assistant Professor \\ Dept. of E\&TC \\ GF's GCOE, Jalgaon, India
}

\author{
K.P. Rane, $\mathrm{PhD}$ \\ Professor \& Head \\ Dept. of E\&TC \\ GF's GCOE, Jalgaon, India
}

\begin{abstract}
Now-a-days every institute needs automation. Automatic Voice Responding system (AVRS) is an automated communication system allowing parent to interact with a student database to know the defined result without using man power. The parent can get the information according to their free time from anywhere by dialing the specified mobile number. The AVRS uses the data stored in database. The parent's mobile number is stored in the database and one mobile number of colleges is given to parent. When a parent dial the mobile number given by college, parent will get the student percentage attendance in the form of voice. So parents can get the student academic performance report from anyplace at their free time. For implementing this AVRS, we are using ARM 11 microcontroller Raspberry Pi and GSM module. The system programming is done by using python. AVRS is specially designed for parent to obtain the student academic performance and the system is highly reliable and scalable. Also the system made user friendly and operating on a $24 * 7$ hour basis. Parent can obtain real time information of student using just mobile phone.
\end{abstract}

\section{Keywords}

Automatic Voice Responding System (AVRS), Raspberry Pi, GSM

\section{INTRODUCTION}

In today's fast life, parents do not have time to visit the school or college to meet teaching faculty to know the student's performance and attendance daily or even weekly too. Also some schools or colleges provide the user name and password to the parents to see their son or daughters academic performance. But this system also requires internet facility. In fact, not every parent can able to operate internet sometime. Hence the automatic voice responding system is used. This system uses the student's data stored in database and parent's number register to the college. Student's data like roll number, name, branch, year and overall percentage attendance is stored in database. Also parent's mobile number is registered to the college database. When the parents call from the registered mobile number to the specified college number, the parents will get the student overall attendance in percentage in form of voice.

The proposed system here can is especially utilized for educational institute like school or college. The system is developed by ARM 11 microcontroller i.e. Raspberry Pi and GSM module. GSM is mostly used among the digital wireless telephony technologies. The GSM module equipment has a Subscriber Identity Module (SIM) security and authentication. The SIM is a data base smart card containing the user's subscription information and phone book. The advantage of the GSM is its international roaming capability in over 100 countries, battery life is improved, efficient spectrum is used, advance features such as short messaging and caller ID, different variety of handset and accessories are used. It has high stability mobile fax and data rate up to 9600 baud.

The goal of our proposed system is to easily get the student's performance and attendance in school or college to follow up whether their child is attending lectures properly or not; from anywhere at any time without human interaction. Parent can get the real time information of academic performance of their son or daughter. The system is made user friendly and reliable and operating on $24 * 7$ hours basis. AVR system provides the quick information to the parent by simply dialling a specified mobile number without visiting to the college.

\section{RELATED WORK}

In 2006, Aysha Qaiser and Shoab A Khan implement the automation of time and attendance using RFID systems [1]. Student and faculty are provided with RFID tags. The system is capable of marking attending, marking unauthorized entry, submission of warning via E-mails, SMS to keep them updated about their child's progress in institute, a dedicated website for availability of the processed data for user. Whenever a warning is submitted at student account in case of on-probation or in case of low weight age, an e-mail containing the same warning is send to the parent or an SMS is sent to parents. Also, if parent wish to see the student's performance; parents has to visit the dedicated website with login in to the system.

Sameeh Ullah and Fakhri Karray in 2008, describes a speaker independent accent based natural language call routing system [2]. This system directs the customer calls to the automatic speech recognition system that is suitable to recognize the input query. Accent identification is required for improving the performance of the natural language call routing system. For this clustering algorithm is used in which a distance metric learning approach, data points are transferred to a new space where Euclidean distance between similar and dissimilar points are at their minimum and maximum respectively. Hybrid clustering approaches improve the performance of the accent classification module in IVR systems.

In 2009[3], Chang-Xing QI, Qing-Dong Du represented a smart IVR system based on application gateway. An IVR system that brings the interactive information by application gateways that communicates between the call center and business information system. This system is based on the function of traditional call center. The application gateway and the IVR system and the information system background resolve the question of format conversation among the various systems in company.

Chang-Xing Qi, Jie Liu, Hang Li in 2010, describes the statistical regression algorithm. In this on the number of node visited it generates the dynamic menu of the IVR [4]. The voice files of data demanded are built and played for response 
through application gateway, TTS and IVR system together. To improve the service and market capacity, modern enterprises are using call center. As a part of call center, the IVR system can bring customers data voices and relevant information by pressing the keys on the normal telephones and entering the required request information. By entering the required information, the IVR will visit the resource of the call center and retrieve the required data information and send the query result to the IVR system and build the voice files by TTS. And this is played to the customer through telephone or sends a text files by fax.

Mudili Soujanya and Sarun Kumar developed the personalized IVR system in contact center in 2010[5]. To develop this speech application software development toolkit used to provide an efficient speech interface, asp.net to supply the requested information to the Microsoft server and interact with the database to process the request of the customer. This is used to provide the service to the customer in accost effective manner without discarding the customer. There are two types of IVR system used touch tone and speech enabled. Touch tone IVR provide the service by having callers select from a list of options using touch tone keypad and speech enable IVR system provide service by having caller select from a list of option by speaking an option or by speaking a keyword.

In 2010, Shanchuan Xu, Wanlin Gao, Zhen Li, Shuliang Zhang, Jianing Zhao proposes a hierarchical system of reusable IVR[6], which reduces the dependence between models and uses XML files as configuration file to make the IVR easily change by modifying the XML file. The new IVR system design which makes IVR system independent from requirements and become a reusable, configurable system as a module of call center.

Comparison of touch and speech-enabled IVR system in low literacy user[7] given by T. jama Ndwe, Mqhele Dlodlo, Jeffery Nichols in 2010 address the feasibility of using the telephone as a tool for information access in the technology challenged and illiterate communities of south Africa. An IVR system is designed for delivering care giving health information using a telephone. This used a DTMF and speech enabled IVR system.

In 2012, Seema Mishra, Apeksha Chavan and Swapnil Gourkar proposed an interactive voice responding system for educational institute [8]. This system uses the pre-recorded information or computer generated voice response to provide information in response to an caller. The input may be given by touch tone or by the dual tone multi frequency signal.

In 2012[9], Santosh Kulkarni and A.R. Karwankar designed a IVRS for college automation which uses the DTMF signal which generates when a caller presses a key of telephone set. This system uses the geortzel algorithm. When a caller dials the number then the technique used for identifying frequency components of a signal is goertzel algorithm. It is digital signal processing technique for identifying frequency component of a signal.

Dusan D. Grujic, Aleksandar R. Milic, Jovana V. Dadic proposed application of IVRS in e-learning system in 2012[10]. In this an interactive voice response solution is used within system for e-education. One of the methods used is open source system for learning the process management is modular object oriented development learning environment. And the system is IVR e-learning which is based on voice synthesis. A speech synthesis means artificial generation of human voice. A computer system used for this purpose is called voice synthesizer and it can be implemented in a suitable hardware or software.

In 2012, A comprehensive study of design, development and implementation of an automated IVR systems [11] is proposed by Mr. Ritesh Chauhan, Mr. Vivek Joshi. This system serves the bridge between people and computer by connecting the telephone network with instructions. The IVR uses the pre-recorded or computer generated voice response to provide information in response to an input from the telephone caller. The input may be in terms of touch tone or DTMF signal, which is generated when a caller press a key on telephone.

T.J. Ndwe, Etienne Barnard describes that the access to information and communication is one of the most important need in any population group in 2013 in correlation between rapid learn ability and user preference in IVR systems for developing regions[12]. This retrains to those who are either poor or illiterate or without having knowledge of computer. Hence the user choice of interaction modality between dual tone multi frequency and speech enabled IVR modalities and correlated the results with learn ability of the different modalities.

\section{PROBLEM STATEMENT}

Existing system are based on DTMF signaling, speech synthesizer, goertzel algorithm. Some system provides the user name and password and by visiting to particular website and entering the required field parents can see the student's performance. Some school or colleges directly calls the parents meeting by sending letter to them. But many times letter doesn't reach home or it reaches late at home or they used to send directly student's progress card. But student doesn't show this at home. Hence parents are not getting the report of student's academic status. So we have tried here to eliminate these drawbacks through our proposed system.

\section{PROPOSED SYSTEM}

The system proposed is basically fully automatic system for college automation and parent convenience. It consists of ARM 11 microcontroller i.e. Raspberry Pi board B+ module and GSM module. The proposed system is based on Linux operating system. Python programming is used to develop the system.

In this system, GSM is used as a mobile phone in college. One number is given to parent of students which is inserted in GSM module. The students' data is stored in database. Student data stored is student name, student id, student mobile number, student branch and overall percentage attendance of the student in academic. Whenever parents call from the registered mobile number in student database to the college number given to parents which is in GSM module, the system will detect the number whether it is registered or not. If they get registered mobile number, the parents are able to hear the student overall attendance in academics and then call will disconnected automatically. The system will automatically respond to the call and parents will get the student attendance in college. If the call coming from the number which is not registered in student database, then the call will be rejected automatically. They are not able to hear any information of the students. Figure 1 shows the overall architecture of the proposed system. 


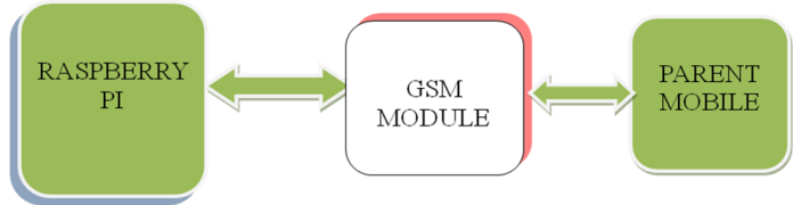

Figure 1: Overall Architecture of Proposed System

\section{ALGORITHM AND FLOWCHART}

\subsection{Flowchart}

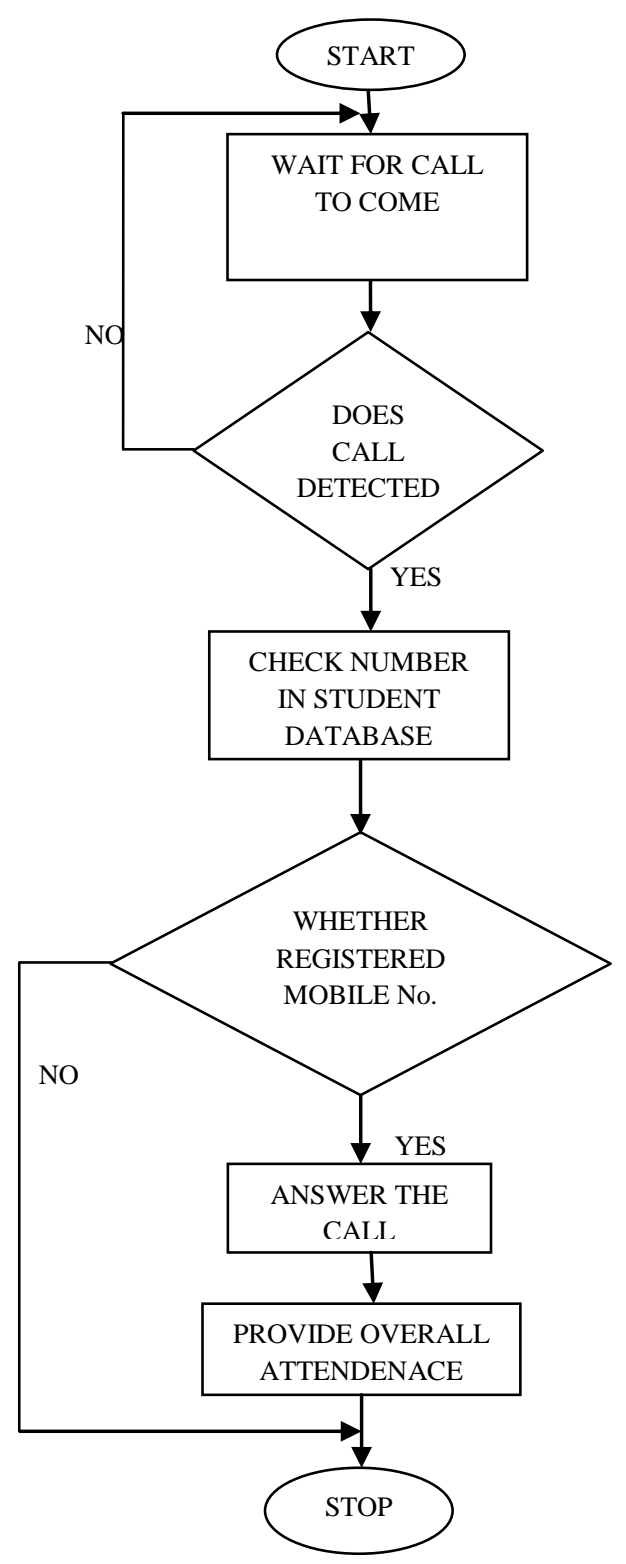

\subsection{Algorithm}

1. Start.

2. Wait for incoming call.

3. MySQL is leading open source database management system. MySQL database is stored in raspberry pi module. We have stored the database of student about student name, student id, student mobile number and student attendance. The information of any student is fetch from student database as per respective call comes and information about attendance of respective student is given to parents. MySQL database is more useful to stored information as compared to the array. MySQL is multiuser, multithread database management system.

4. As parents call from registered mobile number, mobile number of student is verified.

5. If mobile number matches with stored mobile number then call will be responded automatically. For example ATA command is used for receiving the call.

6. If the mobile number doesn't matched with the stored database, the call will be automatically rejected. For example ATH command is used for disconnecting the call.

7. Same process will be repeated for all the incoming call.

8. End

\section{EXPERIMENTAL RESULTS}

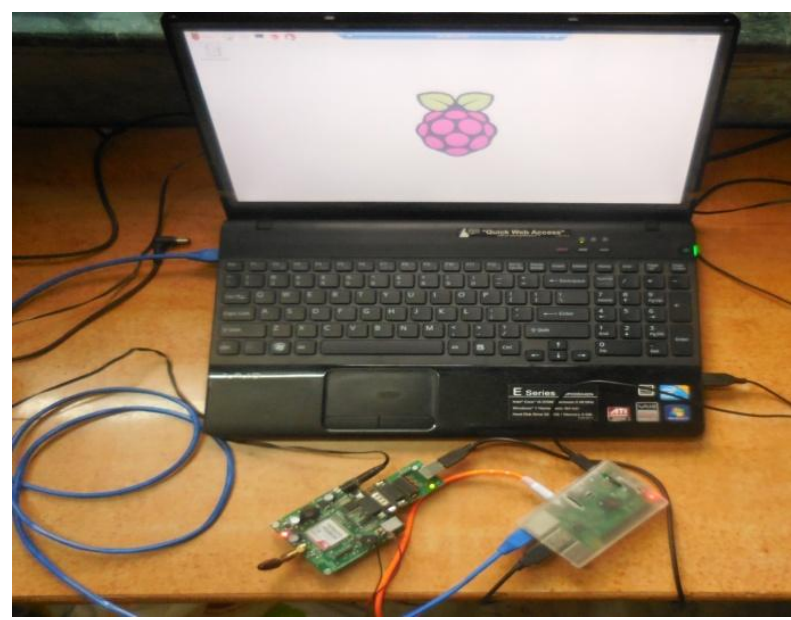

Figure 2: Hardware Implementation of the System

Figure 2 shows the complete hardware of automatic voice responding system for parent of students. It is based on Linux operating system. The Raspberry Pi ARM11 microcontroller plays a vital role in overall system operation. GSM module is interfaced with the microcontroller to detect the mobile number of incoming call. Once the number is detected, microcontroller fetches the data of respective student and through GSM module it is given to the parent in voice form.

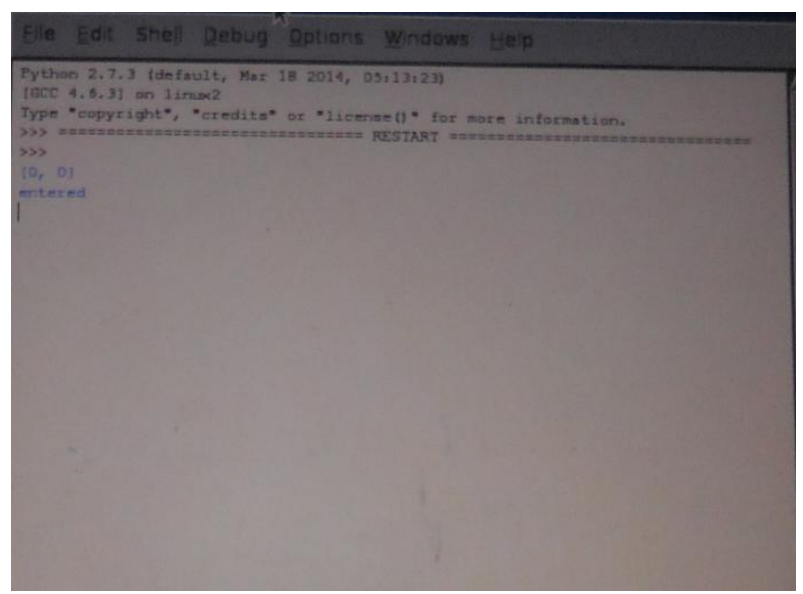

Figure 3: System entered the database 
Figure 3 shows the system is entered in the database and ready to accept the call from the registered mobile number.

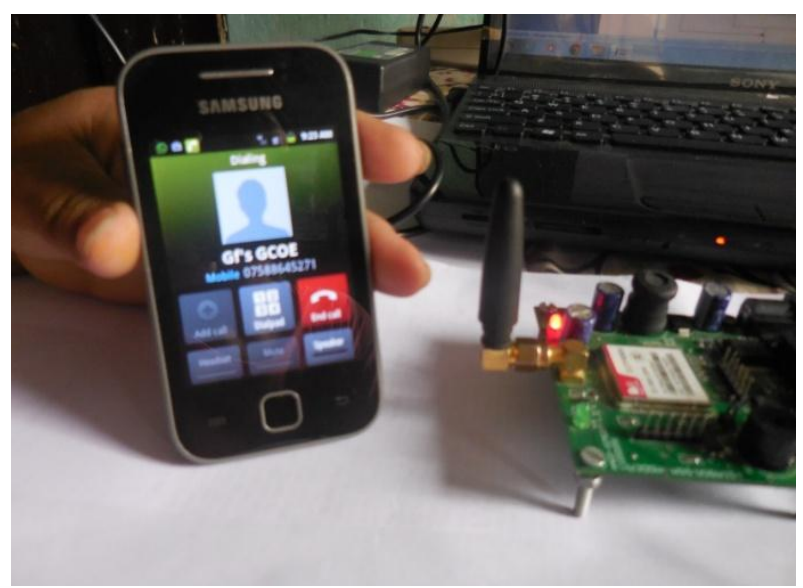

Figure 4: Parent calling to college phone

Figure 4 shows that the parent is calling to the college number from their mobile. After the call is connected the parent mobile number is verified by the micro-controller. If the parent number is registered, call will be accepted otherwise it will be rejected. Here the mobile number of parent is registered to the college. Hence call is accepted.

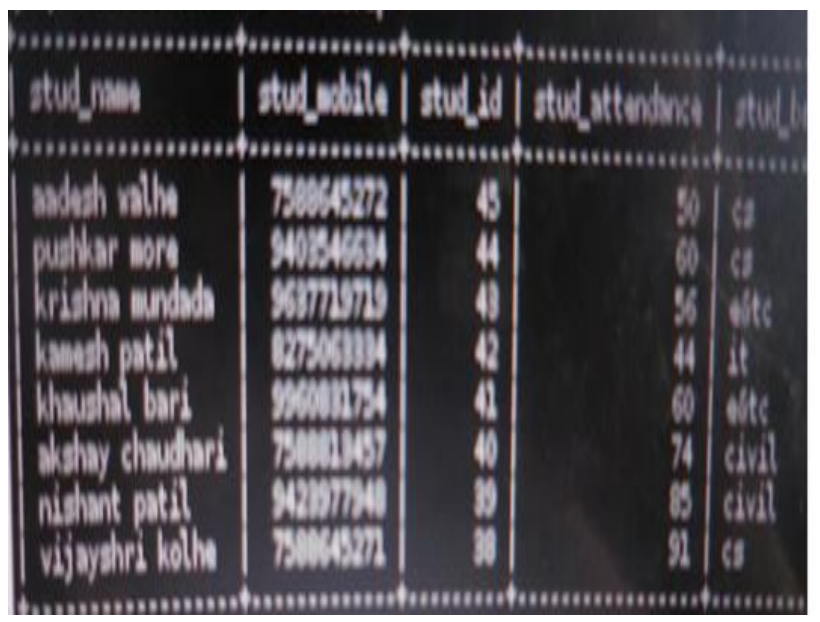

Figure 5: Student Database

Figure 5 shows the student database. The student information is stored in the database. The database contain student name, mobile number, roll number, student overall percentage attendance and branch. The mobile number from which call is coming as shown in Figure 4 is 7588645272 which are present in student database. This mobile number is of Aadesh parents from computer branch. Hence call is accepted.

Figure 6 shows that system has received the call of parent of student named Aadesh Walhe. They get the overall attendance in percentage form as 50\% and the Aadesh Walhe is from computer engineering branch. Parallely the parent can hear the same information through their mobile. This happens for every call of the parent whose mobile number is registered in college. If the mobile number is not registered in the college, the system will automatically reject the call.

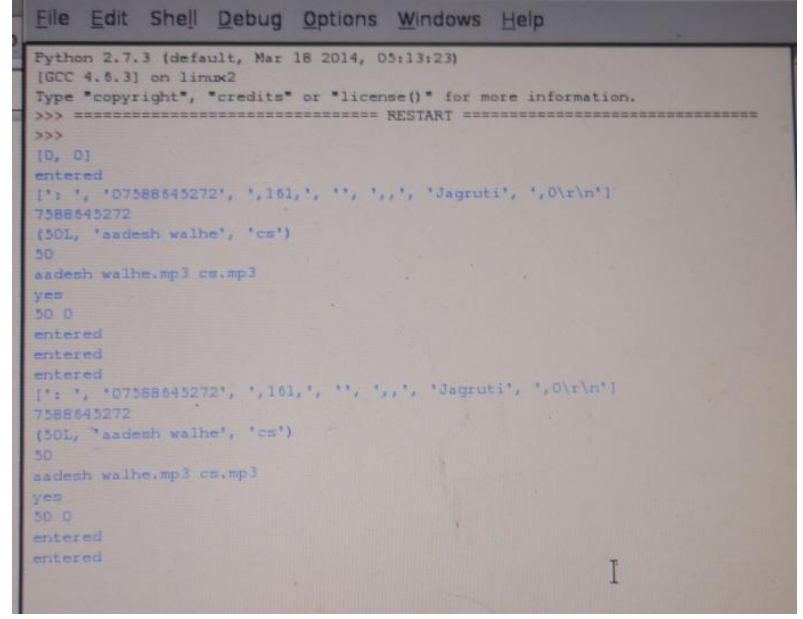

Figure 6: System received the call

\section{CONCLUSION}

Different voice responding techniques in various fields has been reviewed. Every system has its own importance. This project, automatic voice responding system in educational institute for parents of students is implemented using the Raspberry Pi and GSM module. AVRS informs the student's performance in institute to the parents without visiting the college and without human interaction. By using this system it is convenient to get the student details easily to anyone and at any time. By using the Raspberry $\mathrm{Pi}$, there is scope to optimize different methodologies in college automation to make system more users friendly and wide application areas. In future this system can be modified to give test alert information, subject wise marks and test marks are provided to the parents. And SMS will be send to the number from which call has came.

\section{REFERENCES}

[1] Aysha Qaiser and Shoab A Khan, "Automation of time and attendance using RFID systems," IEEE-ICET $2^{\text {nd }}$ international conference on emerging technology, $\mathrm{pp}$ : 61-63, 13-14 Nov. 2006.

[2] Sameeh Ullah and Fakhri Karray, "An evolutionary approach for accent classification in IVR systems," IEEE international conference on systems, man and cybernetics pp: 418-423, 2008.

[3] Hang-Xing QI, Qing-Dong Du, "An smart IVR system based on application gateways," ninth international conference on hybrid intelligent systems, pp: 110-115, 2009.

[4] Chang-Xing Qi, Jie Liu, HangLi, "Application research of statistical regression algorithm in the IVR system," international conference on educational and network technology pp: 358-360, 2010.

[5] Mudili Soujanya, Sarun Kumar,"Personalized IVR system in contact center," internal conference on electronics and information technology, vol 1, pp: 453457,2010

[6] ShanchuanXu, Wanlin Gao, Zhen Li, Shuliang Zhang, Jianing Zhao, "Design of hierarchical and configurable IVR system," $2^{\text {nd }}$ intern. conference on computational intelligent and natural computing, pp: 205-208, 2010. 
[7] T. jama Ndwe, Mqhele Dlodlo, Jeffery Nichols, "Comparison of touch and speech-enabled IVR systems in low literacy users," international conference on user science engineering, pp:244-249, 2010.

[8] Ms Seema P Mishra, Ms Apeksha S. Chavan, Swapnil S. Gourkar, "Interactive voice response system for educational institution, International Journal of Advanced Engineering Technology, April 2012.

[9] Santosh A. Kulkarni, Dr. A.R. karwankar, "IVRS for college automation," international journal of advanced research in computer and communication engineering vol. 1, issue 6, August 2012.

[10] Dusan D. Grujic, Aleksandar R. Milic, Jovana V. Dadic, Marijana S. Despotovic-Zrakic, "Application of IVR in e- learning system," $20^{\text {th }}$ telecommunication forum TELFOR, pp: 1472-1475, 20-22 Nov. 2012.

[11] MR. Ritesh Chauhan, Mr. Vivek Joshi, Prof. Aanchal Jain, "A comprehensive study of design, development and implementation of an automated IVR systems," international journal of computer science and information technology and security vol. 2, no. 6 , December 2012.

[12] T.J.Ndwe, Etienne Barnard, Thato Foko, "Correlation between rapid learn ability and user preference in IVR systems for developing regions," internal information management corporation, 2013. 